\title{
Barreras de acceso al medio físico de los estudiantes con discapacidad motora de la Universidad de Granada
}

\author{
Ma Tamara Polo Sánchez \\ M $^{\text {a Dolores López Justicia }}$
}

Dpto. de Psicología Evolutiva y de la Educación, Universidad de Granada

Spain

tpolo@ugr.es

dlopezj@ugr.es 


\section{Resumen}

Introducción: Desde hace unos años estamos asistiendo a la progresiva incorporación de estudiantes con discapacidad a la universidad. Existen publicaciones (Alcantud, 1995) en las que se narran experiencias de integración social y educativa de los discapacitados en primaria y enseñanza secundaria. No obstante, entraña mayor dificultad encontrar documentación sobre la problemática de los afectados por discapacidad motora en los estudios superiores, lo que justifica que en el presente trabajo se aborde la relevancia de la misma en su proceso de integración.

Método: El trabajo presenta un estudio centrado en conocer qué barreras físicas encuentran 23 estudiantes con discapacidad motora de la Universidad de Granada. Para ello se ha empleado la Escala de Transición de los Minusválidos Físicos y Sensoriales al Mundo Laboral (I.D.E.O, 2003).

Resultados: Los resultados obtenidos muestran que más del 50\% de estos universitarios consideran que son adecuados los servicios y los accesos a las distintas dependencias de los centros, aunque el $47,8 \%$ expresa que no se respetan las plazas reservadas para minusválidos en el aparcamiento.

Conclusión: La Universidad de Granada ha mostrado una gran sensibilidad hacia las personas discapacitadas matriculadas en la misma. Asimismo se han incentivado medidas que contribuyen a la eliminación de barreras de acceso a los servicios, al menos en el caso de los participantes que asisten a las facultades señaladas en este estudio. No obstante, los datos deben ser interpretados con cierta cautela, puesto que aluden a un pequeño porcentaje de población universitaria afectada por discapacidad motora. En este sentido, se acentúa la necesidad de realizar estudios en esta dirección, con objeto de corroborar o no los resultados obtenidos.

Palabras Clave: Discapacidad motora, Universidad, Barreras, Estudiantes universitarios 


\section{Introducción}

En los albores del siglo XXI las personas con discapacidad motora y sus familias continúan en la lucha por combatir los diferentes obstáculos que impiden su desarrollo social integral. Para lograr la equidad en la diversidad han tenido que salvar enormes dificultades que van desde enfrentarse a barreras físicas y arquitectónicas e incluso a barreras culturales.

La mejor forma de constatar y evidenciar las dificultades de movilidad y accesibilidad de estas personas, es hacer un recorrido breve en su compañía, para así ir evidenciando y anotando cada uno de los obstáculos encontrados. En este sentido, el análisis de los diferentes tipos de barreras arquitectónicas nos permite distinguir entre:

- Barreras arquitectónicas urbanísticas: son las que se encuentran en las vías y espacios públicos, aceras, pasos de distinto nivel, obstáculos en la vía pública, parques y jardines no accesibles.

- Barreras arquitectónicas en la edificación: son las que se encuentran en el interior de los edificios, escalones, peldaños, pasillos, ascensores reducidos, etc.

- Barreras en el transporte: son aquéllas que se encuentran en los diferentes medios de transporte, inaccesibilidad al transporte público, dificultades para el estacionamiento del transporte privado.

A partir de esta descripción es posible tener una visión general de la problemática a la que se enfrenta cualquier persona con discapacidad motora a la hora de sortear las múltiples barreras arquitectónicas que se le presentan a diario, siendo fundamental que se sienta la necesidad de constatar estas dificultades para evidenciarlas y tratar de suplirlas en su momento. Se sabe que las barreras arquitectónicas condicionan su proceso de integración, representan un aspecto central en la búsqueda de mejorar la calidad de vida de estas personas, restringen el acceso a los espacios para el desempeño de actividades de la vida cotidiana, limitan el desarrollo de sus aptitudes personales y reducen su participación en la vida social y productiva; todo ello justifica la conveniencia y la necesidad de suprimirlas (Guzmán, 2001). 
Pero la tarea de eliminar barreras no es nada simple e implica un largo proceso de sensibilización e información a la población con y sin discapacidad, además de la capacitación constante de los diferentes servidores públicos.

En repetidas ocasiones se ha argumentado que una forma de favorecer la integración de las personas con discapacidad motora pasa por desarrollar su competencia en el terreno formativo. Por ello, no es extraño encontrar cada día más estudiantes universitarios con esta problemática. Pero acceder a los estudios universitarios no es fácil para personas con discapacidad, requiriendo una gran fuerza de voluntad, el uso de numerosos recursos personales, técnicos, económicos y el apoyo incuestionable de sus familiares y amigos (Alcantud, 1995).

Entre las dificultades que con mayor frecuencia presentan los estudiantes matriculados en las universidades españolas se encuentran los problemas de tipo motor y de desplazamiento. Esto se confirma también en la Universidad de Granada pues, si tomamos en consideración algunos datos recogidos por el Gabinete de Atención Social, durante el curso académico 2003/04 se matricularon 287 alumnos que presentaban alguna discapacidad; de ellos un $58,9 \%$ (169) estaban afectados de discapacidad física, un 13,6\% (39) de discapacidad sensorial auditiva y un $17,1 \%$ (49) presentaba discapacidad sensorial visual. Por último, un 3,5\% (10) déficit psíquico.

Como consecuencia de la presencia de alumnado con discapacidad motora en los centros universitarios españoles, se ha prestado atención a sus problemáticas y se ha potenciado la investigación sobre aspectos como las barreras de accesibilidad. De hecho, un alto porcentaje de universidades dedican desde hace tiempo una atención preferente a los alumnos y ofrecen programas para garantizar su acceso a los estudios superiores y facilitar su integración en la vida profesional. A pesar de que es todavía escaso su acceso a la Universidad, no obstante cada año se superan más trabas en ese camino hacia la igualdad de oportunidades. Algunas de las experiencias realizadas en diferentes universidades españolas, abordando la eliminación de barreras, se señalan a continuación:

De la Red, de la Puente, Gómez y Carro (2002) llevaron a cabo un programa centrado en el proceso de atención a las personas con discapacidad física y sensorial en la Universidad de Valladolid, consistente en la puesta en práctica de una serie de gestiones con la intención de facilitarles la integración en el contexto universitario. Entre dichas medidas se incluía el 
apoyo en la solicitud de eliminación de barreras arquitectónicas para garantizar el fácil acceso, puesto que aparecían determinadas limitaciones en el ambiente de accesibilidad, infraestructura, comunicación y organización que perjudican el normal acceso a los estudios universitarios.

De manera similar, en la Comunidad de Madrid se llevó a cabo una investigación para conocer la situación de acceso e integración de estudiantes discapacitados, con la finalidad de dotarles de un instrumento útil y proporcionar, a las instituciones competentes, un documento de reflexión. Entre las propuestas de mejora se incluía como objetivo general la necesidad de garantizar a los alumnos y alumnas con necesidades educativas específicas el acceso a la Universidad y la continuación de sus estudios en igualdad de oportunidades con los demás estudiantes; todo ello mediante la adecuación de los centros universitarios para facilitar el acceso a las instalaciones eliminando las barreras arquitectónicas y urbanísticas y dotando a los centros de equipamientos especiales y apoyos pedagógicos (Touza, 2000).

También se ha avanzado, aunque tímidamente, en la atención a la integración laboral de estudiantes con discapacidad, como lo refleja el estudio de Campoy y Pantoja (2003). En éste pusieron de manifiesto que, en el contexto actual, las personas con discapacidad tienen mayores dificultades para encontrar empleo que la población general pues, además de su propia minusvalía, deben superar barreras arquitectónicas y barreras psicosociales.

Ante la escasa documentación encontrada que aborde esta temática y la existencia de un buen número de estudiantes universitarios granadinos afectados por discapacidad motora, nos planteamos la puesta en práctica del presente estudio, el cual pretendía conocer qué barreras físicas encuentran estos estudiantes en el entorno universitario. Somos conscientes de que será a partir del análisis de sus necesidades cuando se podrán adoptar las medidas oportunas que incidan favorablemente en su integración personal y social.

\section{Método}

La metodología de investigación seleccionada para la puesta en práctica de este trabajo fue descriptiva, basada en la aplicación de un cuestionario como forma de recogida de información, ya que el principal fin era describir sistemáticamente hechos y características de una población dada o área de interés de forma objetiva y comprobable. 


\section{Sujetos}

Durante el curso 2003/04 se matricularon en la Universidad de Granada 169 alumnos y alumnas que presentaban discapacidad motora. Aunque la pretensión en esta investigación era llegar al total de esta población, hay que indicar la gran dificultad para acceder a la totalidad de los sujetos por diferentes motivos derivados de la idiosincrasia de su problemática. Del total de estos 169 alumnos y alumnas con discapacidad motora sólo dieron respuesta a la escala 23 de ellos.

Los rasgos o patrones identificadores de esta muestra se detallan a continuación:

- $\quad$ Se trataba de un grupo compuesto mayoritariamente por hombres discapacitados, $60,9 \%$ (14) frente a un 39,1\% (9) de mujeres discapacitadas.

- El 8,69\% (2) de los estudiantes presentaba acondroplasia, el 34,78\% (8) afección en miembros superiores, el 43,47\% (10) en miembros inferiores. Un 13,04\% (3) padecía alguna afección tanto en miembros superiores como inferiores.

- $\quad$ El 65,2\% (15) de los encuestados tenía una edad superior a los 24 años, mientras el $34,8 \%$ (8) tenía una edad inferior a la misma.

- $\quad$ Generalmente eran solteros/as, el 87\% (20). Tan solo uno era casado 13\% (3).

- Existía un predominio mayoritario de afectados por discapacidad motora que se dedicaban exclusivamente al estudio (82,6\%/19), mientras que el $13 \%$ (3) compaginaba estudio y trabajo.

- Entre los lugares de residencia destacaba Granada capital (65,2\%/15), le seguían los pueblos cercanos a Granada $(30,4 \% / 7)$, tan solo un participante no contestaría a dicha cuestión.

- En relación al transporte de uso común, el 43,5\% (10) hacía uso del transporte público, el $43,5 \%$ usaba coche privado (10), el 26,1\% (6) iba a pie. Ninguno utilizaba vehículos del organismo al que pertenecen como personas que padecen una minusvalía. 
- Entre los estudiantes que cumplimentaron el cuestionario, era destacable la mayor participación de los procedentes de la licenciatura en Psicología $(21,7 \%)$, seguidos de la especialidad en Magisterio de Educación Primaria (8,7\%), Magisterio de Educación Especial (4,3\%), Audición y Lenguaje (4,3\%), Empresariales-Economía (4,3\%), Medicina (4,3\%), Informática (4,3\%), Bellas Artes (4,3\%), Sociología (4,3\%), Farmacia (4,3\%), Ciencias de la Actividad Física y el Deporte (4,3\%), Odontología (4,3\%), Física (4,3\%), Psicopedagogía (4,3\%), Filosofía (4,3\%) y Educación Social (4,3\%).

\section{Instrumento}

El instrumento de análisis empleado fue la Escala de Transición de los Minusválidos Físicos y Sensoriales Universitarios al Mundo Laboral (Grupo de Investigación y Desarrollo Educativo de la Orientación, 2003). Dicha escala se concibió para la aplicación a estudiantes universitarios discapacitados y trabajadores discapacitados con titulación universitaria. Con tal fin se incluyeron los siguientes apartados a cumplimentar: variables de identificación (12 ítems); variables físicas en el lugar de trabajo (10 ítems) y barreras físicas en el lugar de estudio (8 ítems); variables socio-personales (19 ítems); y variables socio-profesionales (11 ítems). Las respuestas a cada uno de los apartados corresponden a la percepción que se tiene sobre las distintas variables en función de la siguiente escala: 1 , muy de acuerdo; 2 , de acuerdo; 3 , en desacuerdo. Se seleccionó porque había sido utilizada en investigaciones similares, e incluye ítems que evalúan específicamente barreras físicas, que constituían el núcleo central de estudio.

Campoy y Pantoja (2003), en una investigación llevada a cabo en la Universidad de Jaén, comprobaron la validez y la fiabilidad de esta escala. Comprobaron la validez desde el punto de vista del contenido y en función de los resultados procedieron a la aplicación definitiva de la escala. El índice de homogeneidad de cada ítem se entendió como un coeficiente de correlación entre el ítem y el resto de la escala. El coeficiente de fiabilidad se obtuvo mediante el coeficiente alfa de Cronbach $(\mathrm{alfa}=.8460)$.

\section{Procedimiento}

La primera aproximación a la población de estudiantes universitarios con discapacidad motora, supuso el contacto con el Gabinete de Atención Social de la Universidad de Granada (concretamente con el personal a cargo del "Programa de Intervención Social hacia Estudiantes con Discapacidades"), dado que desde el curso 1992/93 la Universidad de Granada, a 
través del Vicerrectorado de Estudiantes, lleva realizando actuaciones con los estudiantes con discapacidad con el fin de facilitar su inserción y participación en la Universidad.

Los estudiantes fueron informados mediante una carta del sentido del trabajo y se les citó para explicarles de forma pormenorizada todos los detalles del estudio. A continuación se les solicitó la participación y que cumplimentaran la Escala de Transición de los Minusválidos Físicos y Sensoriales Universitarios al Mundo Laboral.

A pesar de que la reunión informativa se llevó a cabo en horario de mañana y de tarde para que asistieran el mayor número posible de alumnos y alumnas, fue necesario convocar otra nueva sesión ante la demanda de los propios estudiantes que no pudieron asistir a la primera.

No fue necesario someter la escala a ningún tipo de modificación ya que los estudiantes tenían buena movilidad manual, excepto en un caso, en el cual la ausencia de miembros superiores motivó que la propia investigadora tuviera que prestar ayuda para poder rellenar el cuestionario.

\section{Resultados}

Para la estructuración, ordenación y análisis de los datos se ha utilizado el paquete de programas estadísticos SPSS en su versión 12 para Windows, que por su versatibilidad y fiabilidad de uso parece especialmente indicado para investigaciones de tipo descriptivo. La evaluación que los alumnos/as hacen en términos porcentuales de las características físicas del lugar de estudio se presenta a continuación y se refleja en la tabla 1. 
Tabla 1. Barreras físicas del lugar de estudio.

\begin{tabular}{|l|c|c|c|c|}
\hline & No contesta & $\begin{array}{c}\text { Muy de } \\
\text { acuerdo }\end{array}$ & De acuerdo & En desacuerdo \\
\hline El acceso a la biblioteca me parece & 2 & 11 & 4 & 6 \\
adecuado & $8,7 \%$ & $47,8 \%$ & $17,4 \%$ & $26,1 \%$ \\
El acceso al salón de actos me & 2 & 11 & 8 & 2 \\
parece adecuado & $8,7 \%$ & $47,8 \%$ & $34,8 \%$ & $8,7 \%$ \\
La cafetería tiene buenos accesos & 2 & 14 & 4 & 3 \\
& $8,7 \%$ & $60,9 \%$ & $17,4 \%$ & $13,0 \%$ \\
Hay WC habilitados para & 3 & 8 & 6 & 6 \\
minusválidos & $13,0 \%$ & $34,8 \%$ & $26,1 \%$ & $26,1 \%$ \\
Resulta fácil el acceso a los & 2 & 9 & 8 & 4 \\
mostradores de información & $8,7 \%$ & $39,1 \%$ & $34,8 \%$ & $17,4 \%$ \\
Las aulas permiten seguir las & 2 & 7 & 8 & 6 \\
clases con comodidad & $8,7 \%$ & $30,4 \%$ & $34,8 \%$ & $26,1 \%$ \\
El transporte público se adecua a & 4 & 6 & 6 & 7 \\
mis necesidades & $17,4 \%$ & $26,1 \%$ & $26,1 \%$ & $30,4 \%$ \\
Se respetan las plazas reservadas & 5 & 4 & 3 & 11 \\
para el aparcamiento & $21,7 \%$ & $17,4 \%$ & $13,0 \%$ & $47,8 \%$ \\
\hline
\end{tabular}

Respecto de las barreras de acceso a la biblioteca. El 47,8\% (muy de acuerdo) y el $17,4 \%$ (de acuerdo) considera que el acceso a la biblioteca es adecuado a su discapacidad, mientras el 26,1\% manifiesta su desacuerdo.

- En el ítem de acceso al salón de actos, al igual que ocurría en el ítem referido al acceso a la biblioteca, existe una amplia mayoría $(47,8 \%$ y $34,8 \%)$ a la que le resulta muy adecuado el acceso.

Acceso a la cafetería. La entrada a la cafetería es otro de los aspectos frente a los cuales los universitarios discapacitados expresan su acuerdo (60,9\% muy de acuerdo/ 17,4\% de acuerdo).

- Habilitación de servicios sanitarios. Existe un porcentaje elevado (34,8\%) de estudiantes universitarios discapacitados que demandan mayor adecuación de los servicios para minusválidos.

Acceso a los mostradores de información. El 39,1\% de los estudiantes universitarios granadinos (muy de acuerdo) y 34,8\% (de acuerdo) consideran que les resulta fácil el 
acceso a los mostradores informativos de la Facultad, mientras que el 17,4\% considera inadecuado el paso hasta los mismos.

Adecuación de las aulas. El 26,1\% expresa que la disposición de las aulas no les permite seguir las clases con comodidad; el 30,4\% (muy de acuerdo) y el 34,8\% (de acuerdo), en cambio, afirman que las aulas sí son adecuadas para seguir el desarrollo de las clases.

Adecuación del transporte público. El 26,1\% (muy de acuerdo) y 26,1\% (de acuerdo) de los estudiantes expresa que el transporte público se adapta a sus necesidades, el $30,4 \%$ demanda una mejora de este tipo de transporte.

Plazas de aparcamiento. En cuanto a las plazas de aparcamiento, un porcentaje alto $(21,7 \%)$ no contesta a este ítem, hecho que se puede interpretar como una carencia de información o bien a que no utilizan este servicio, al no precisarlo por su propia discapacidad. No obstante, el porcentaje de alumnos que contestan a tal cuestión $(47,8 \%)$ expresa que no se respetan las plazas reservadas para minusválidos en el aparcamiento de la Facultad.

\section{Discusión}

Los resultados obtenidos en el presente estudio nos permiten afirmar que un porcentaje alto de los estudiantes universitarios granadinos afectados por discapacidad de tipo motor están muy de acuerdo o de acuerdo con los accesos a las distintas dependencias universitarias: a la biblioteca, al salón de actos, a la cafetería, a los mostradores de información; consideran adecuada la habilitación de servicios sanitarios, de las aulas y del transporte público. Por otra parte, un buen número de estos estudiantes están en desacuerdo con el uso que hacen los demás de las plazas de aparcamiento reservadas para las personas con discapacidad, pues opinan que no se respeta su uso.

Si bien, de entrada, puede parecer que este último aspecto no es muy importante, no obstante, el uso de los medios de transporte puede tener implicaciones en la vida de la persona discapacitada, tanto en el ámbito educativo como en el laboral, facilitando u obstaculizando su inserción en el mismo. De hecho, Robert, Beadles, Randall \& Stacy (2000), señalaban la necesidad de planificar estudios que permitan hacer frente a aquellos factores asociados con 
los medios de transporte (vehículo propio, vehículo del organismo, reserva en plazas de estacionamiento, etc.) que pueden incidir en mayor o menor grado en la integración social y en el acceso al mundo laboral.

La valoración que hacen los estudiantes universitarios granadinos con discapacidad motora sobre los obstáculos físicos nos permite afirmar la existencia de una analogía con otros estudios realizados en esta vía, especialmente en el caso de las personas que padecen déficit visual (Campoy y Pantoja, 2003; Robert et al., 2000). El presente estudio también coincide con el trabajo de Campoy y Pantoja (2003), realizado en la Universidad de Jaén, en la estimación que realizan del acceso a la biblioteca, salón de actos o mostradores de información, adecuación de las aulas a las necesidades de los estudiantes, considerándose adecuados en su mayoría al tipo de discapacidad; asimismo, los resultados concuerdan en la apreciación de que deberían tomarse medidas adicionales para que se respetaran las plazas de aparcamiento.

A la luz de los datos podemos concluir que la Universidad de Granada ha mostrado sensibilidad hacia las personas con discapacidad matriculadas en la misma y ha tomado medidas que contribuyan a la eliminación de barreras de acceso a los distintos servicios, al menos en el caso de estudiantes que asisten a las facultades señaladas en este estudio. De aquí se desprende su reconocimiento de que estas personas son capaces y contemplan opciones propias de vida, tan legítimas como las de los demás integrantes de la sociedad; hay que aprovechar al máximo sus posibilidades para permitir su integración y participación en la sociedad en igualdad de condiciones y permitir su pleno reconocimiento social, mediante la supresión de obstáculos.

Queremos señalar que una limitación de nuestro estudio está en la reducida muestra que ha participado en el mismo, puesto que los resultados aluden a un porcentaje limitado de la población universitaria granadina con discapacidad motora, por lo que llamamos la atención sobre la conveniencia de diseñar estudios que contemplen la perspectiva de un número mayor de estudiantes, con objeto de corroborar o no los resultados obtenidos.

\section{Referencias}


Alcantud, F. (1995). Estudiantes con discapacidades integrados en los estudios universitarios. Notas para su integración. En F. Rivas, Manual de Asesoramiento y Orientación Vocacional, 455-470. Madrid: Síntesis.

Campoy, T.J. y Pantoja, A. (2003). Transición al mundo laboral de alumnos universitarios con discapacidades físicas y sensoriales. Revista de Educación Especial, 33, 39-57.

De la Red, N., de la Puente, R., Gómez, Mª.C. y Carro, L. (2002). Marco contextual y operativo de las personas con discapacidad en la Universidad. En N. De la Red, R. de la Fuente, Ma . C. Gómez y L. Carro, El acceso a los estudios superiores de las personas con discapacidad física y sensorial, 55-130. Valladolid: Secretariado de Publicaciones e Intercambio Editorial.

Grupo de Investigación y Desarrollo Educativo de la Orientación (2003). Escala de Transición de los Minusválidos Físicos y Sensoriales al Mundo Laboral (no publicado).

Guzmán, A. 2001. Barreras de acceso: Soluciones alternativas en la escuela. Red de inclusión educativa. En http://www.inclusioneducativa.cl.

Robert, J., Beadles, Jr., Randall, M. \& Stacy, M.S. (2000). Vocational Outcomes of Sensory Impaired Graduates of an Adult Vocational Training Program. Journal of Visual Impairment and Blindness, 94 (5), 275-280.

Touza, E. (coord.) (2000). Acceso e integración de estudiantes discapacitados en las Universidades de la Comunidad de Madrid. Madrid: Comunidad de Madrid, Consejería de Educación, Fundación Universidad-Empresa. 